\title{
Prediction of jet mixing noise with Lighthill's Acoustic Analogy and geometrical acoustics
}

\author{
Carlos R. S. llário ${ }^{\text {a) }}$ \\ EMBRAER S.A., Avenue Brigadeiro Faria Lima, 2170, 12227-901, São José dos Campos - SP, Brazil \\ Mahdi Azarpeyvand \\ Department of Mechanical Engineering, University of Bristol, Bristol, United Kingdom \\ Victor Rosa and Rod H. Self \\ Institute of Sound and Vibration Research, University of Southampton, Southampton, United Kingdom \\ Júlio R. Meneghini \\ NDF, Escola Politécnica, University of São Paulo, São Paulo, Brazil
}

(Received 8 August 2016; revised 11 January 2017; accepted 14 January 2017; published online xx Xx Xxxx)

\begin{abstract}
A computational aeroacoustics prediction tool based on the application of Lighthill's theory is presented to compute noise from subsonic turbulent jets. The sources of sound are modeled by expressing Lighthill's source term as two-point correlations of the velocity fluctuations and the sound refraction effects are taken into account by a ray tracing methodology. Both the source and refraction models use the flow information collected from a solution of the Reynolds-Averaged NavierStokes equations with a standard $k$-epsilon turbulence model. By adopting the ray tracing method to compute the refraction effects a high-frequency approximation is implied, while no assumption about the mean flow is needed, enabling the authors to apply the new method to jet noise problems with inherently three-dimensional propagation effects. Predictions show good agreement with narrowband measurements for the overall sound pressure levels and spectrum shape in polar angles between $60^{\circ}$ and $110^{\circ}$ for isothermal and hot jets with acoustic Mach number ranging from 0.5 to 1.0. The method presented herein can be applied as a relatively low cost and robust engineering tool for industrial optimization purposes. (C) 2017 Acoustical Society of America.
\end{abstract}

[http://dx.doi.org/10.1121/1.4976076]

[JWP]

Pages: $1-11$

\section{INTRODUCTION}

Despite great reductions of aircraft noise achieved in the past few decades, the current trend of continuous growth of air traffic worldwide will demand further reduction of noise emission by civil and military aircraft. Due to the inherent complexity of aerodynamic noise generation and propagation phenomena, industrial and academic efforts have been focused on the development of reliable and computationally low-cost noise prediction tools for the aircraft design process. Jet mixing noise is one among the dominant sources of aircraft noise, being more pronounced at take-off condition. As the jet mixing noise has been greatly reduced by increasing the bypass ratio of dual-stream-jet engines, further jet mixing noise reductions are likely to rely on modifications of the nozzle geometry that may result in the use of nonaxisymmetric nozzles and therefore very complex threedimensional flows. For instance, it has been verified both experimentally ${ }^{1-3}$ and computationally ${ }^{4}$ that the use of chevron nozzles and non-concentric dual-stream nozzles can lead to jet mixing noise reduction.

The development of numerical prediction methods for jet noise is perhaps one of the oldest areas of aeroacoustics. Methods ranging from empirical database ${ }^{5}$ to high-fidelity

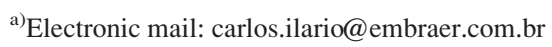

and computationally expensive methods ${ }^{6-8}$ have been con- 49 sidered over the past few decades. Nevertheless, a cheap, 50 fast, and reliable numerical method that provides an accurate 51 prediction is still needed to help the optimization process in 52 an industrial context. The hybrid numerical methodology 53 based on a Reynolds Averaged Navier-Stokes (RANS) solu- 54 tion of the flow presented in this paper is seen as an alterna- 5 tive method to fulfill this requirement.

An early application of such hybrid methodology to compute jet mixing noise was presented by Balsa and Gliebe $^{9}$ and Balsa et al., ${ }^{10}$ who used analytical profiles to describe the mean flow and model the source term of the equation presented by Lilley. ${ }^{11}$ The approach was later extended by Khavaran et al. ${ }^{12}$ and Khavaran and Krejsa ${ }^{13}$ to use a numerical RANS $k-\varepsilon$ solution of the mean flow into the so-called MGBK (Mani, Gliebe, Balsa, and Khavaran) method; thus consolidating the use of a RANS $k-\varepsilon$ and an acoustic analogy to model jet mixing noise.

The idea was further explored by Tam and Auriault, ${ }^{14}$ who modeled the sound sources via an analogy with the kinetic theory of gases. They added the proposed source term to an adjoint formulation of the Linearized Euler Equations, therefore departing from the use of an acoustic analogy; their predictions of far-field sound pressure level (SPL) showed good agreement with measurements. Morris and Farassat ${ }^{15}$ showed that although not explicitly an 
75 acoustic analogy, Tam and Auriault's method is akin to what

76 can be derived from an acoustic analogy, and showed that

77 the improvements by Tam and Auriault's method was the

78 better description of the turbulence statistics relevant for the

79 description of the sources of sound.

80 Self $^{16}$ followed by proposing a model based on

81 Lighthill's Acoustic Analogy (LAA) with an improved

82 description of the relevant turbulence statistics based on

83 empirical evidence by Harper-Bourne. ${ }^{17}$ The main improve-

84 ment was the consideration of frequency-dependent time and

85 length-scales when modeling velocity correlations present in

86 LAA's source term. The proposed model resulted in good

87 agreement with experimental data, notably with a better

88 description of the decay at low and high frequencies when

89 compared to the LAA-based method of Morris and

90 Farassat. $^{15}$ Self and Azarpeyvand ${ }^{18,19}$ and Azarpeyvand and

91 Self $^{20}$ further developed the idea of frequency-dependent

92 scales of velocity correlations by proposing a new time scale

which was applied to the MGBK method.

In this paper a source model based on the LAA with the new time scale of Refs. 18-20 is presented. The resulting statistical source is shown to result in a good description the far-field spectrum at $90^{\circ}$. To overcome the shortcoming of LAA that ignores effects of propagation, a geometrical acoustics approximation is applied. The application of geometrical acoustics is not new in jets, ${ }^{21-23}$ but it is, to the authors best knowledge, for the first time coupled to a source model based on the LAA to predict jet mixing noise instead of just analyze aspects of it. Another way to compute the propagation effects is to solve the adjoint formulation of the linearized Euler equations using a finite difference method (FDM). ${ }^{24}$ Using a FDM, however, increases the computa07 tional cost of the overall prediction method as the FDM is 108 expensive and known to generally require a mesh of higher 09 quality (finer and structured) than the RANS mesh. The ray 110 tracing method used in this paper, in contrast, needs only to 111 interpolate the results from the RANS into a coarser mesh. 112 The main objective of this paper is therefore to introduce 113 and benchmark a novel hybrid aeroacoustics method that can 114 be applied to predict the far-field noise from arbitrary three115 dimensional jets. The method was created with the goal of 116 providing the ability for both the analysis and the optimiza117 tion of nozzles that would be compatible with novel configu118 rations, yet requiring relatively low computational cost.
The remainder of the paper is organized as follows. Section II deals with the source and propagation models developed as part of this work. The experimental setup and solution of the mean flow are presented in Sec. III. Also in Sec. III the far-field noise predictions for jets at different Mach numbers and temperature ratios predicted using the new model will be compared against the available experimental data at different angles. Results will be presented for jet noise prediction at $90^{\circ}$, source distribution, flow factor, and jet noise directivity. Finally, Sec, IV concludes the paper.

\section{MATHEMATICAL MODEL}

The mathematical modeling of the new jet noise prediction tool is provided in this section. The far-field noise can be predicted by coupling the source and propagation models, 132 presented in Secs. II A and II B. The models are derived sep- 133 arately, emphasizing the fact that they are completely inde- 134 pendent and can be used in isolation.

\section{A. Source model}

The starting point of the source model is the Lighthill 137 equation, ${ }^{25}$ as presented by Ribner. ${ }^{26}$ The far field spectrum 138 can be written as

$$
P(\boldsymbol{x} ; \omega)=\frac{1}{(4 \pi r)^{2}} \frac{1}{a_{0}^{4}} \bar{\rho}^{2} D_{f}^{-5} d_{i j k l} \int \Phi \mathcal{F}\left[I_{i j k l}\right] \mathrm{d}^{3} \boldsymbol{y},
$$

where $r=|\boldsymbol{x}|$ is the distance to the far-field observer, and $\boldsymbol{x} 140$ and $\boldsymbol{y}$ are, respectively, the observer and source locations. 141 The coordinate system $(r, \theta, \varphi)$ is shown in Fig. 1. In Eq. 142 (1), $a_{0}$ is the reference speed of sound, $\bar{\rho}$ is the mean fluid 143 density, $D_{f}$ is the Doppler factor $\left(1-M_{c} \cos \theta\right), d_{i j k l}$ is the 144 tensor giving the quadrupolar directivity, $\Phi$ is the flow factor 145 (introduced in the next section), $\mathcal{F}$ denotes the Fourier trans- 146 form, and $I_{i j k l}$ represents the contribution from fourth-order 147 velocity correlations.

The convective Mach number $\left(M_{c}\right)$ is assumed to 149 depend on the local Mach number $\left(U_{1} / a\right)$ and the nozzle exit 150 Mach number $\left(M=U / a_{0}\right)$ and is given by ${ }^{12}$

$$
M_{c}=\frac{1}{4}\left(\frac{U_{1}}{a}\right)+\frac{1}{3} M
$$

where $U_{1}$ is the local mean axial velocity, $U$ the jet-exit 152 velocity, and $a$ the local mean sound speed.

The tensor $I_{i j k l}$ represents the contribution of the fourth- 154 order velocity correlation terms and is given by

$$
I_{i j k l}(\tau)=\int \frac{\partial^{4}}{\partial \tau^{4}} \overline{v_{i} v_{j} v_{k}^{\prime} v_{l}^{\prime}} \mathrm{d}^{3} \xi
$$

where $v_{i}=U_{i}+u_{i}$ is the instantaneous velocity vector, the 156 prime indicates that the property is evaluated at a different 157

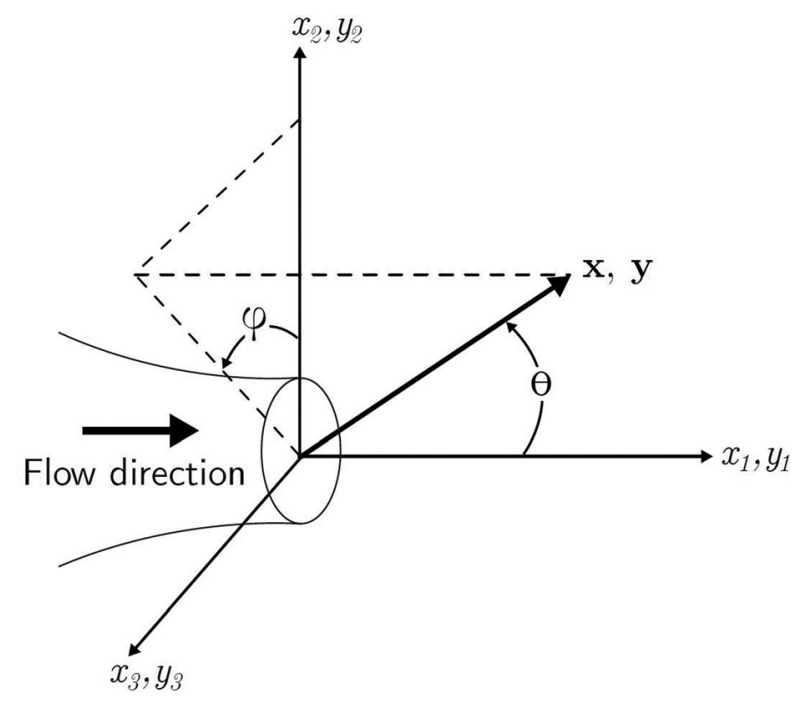

FIG. 1. Cartesian and spherical coordinate systems. 
180 where $f$ is a function of the separation vector $\xi$, and $181 f^{\prime}=d f / d \xi$. Among different possibilities, ${ }^{26} f$ is assumed 182 here to take a Gaussian distribution form

$$
f(\xi)=\exp \left(-\pi \frac{\xi^{2}}{L^{2}}\right)
$$

183 where $L$ is the length-scale at the source location.

With the substitution of Eqs. (8) and (9) in Eq. (7) and performing the integral over the source region $(\xi)$, the term $186 I_{i j k l}$ reduces to

$$
I(\tau)=\frac{\rho^{2}}{2 \sqrt{2}} k^{2} L^{3} \frac{\partial^{4} g^{2}(\tau)}{\partial \tau^{4}},
$$

where $k$ is the local mean turbulent kinetic energy.

Here the directivity index ijkl is dropped to emphasize that the source is isotropic due to the assumption of isotropic turbulence. Thus the far-field directivity is modeled by the convective amplification given by $D_{f}^{-5}$ and refraction (pre- 191 sented in Sec. II B).

It is assumed that the temporal correlation function, $g, 193$ also takes a Gaussian distribution form, as

$$
g(\tau)=\exp \left(-\tau^{2} / \tau_{0}^{2}\right),
$$

where $\tau_{0}$ is the time scale at the source location. Taking the 195 Fourier transform of $\partial^{4} g^{2} / \partial \tau^{4}$ in Eq. (10) leads to

$$
I(\Omega)=\frac{\sqrt{\pi}}{4} k^{2} L^{3} \tau_{0} \Omega^{4} \frac{\sqrt{2 \pi}}{2} \exp \left(-\frac{\tau_{0}^{2} \Omega^{2}}{8}\right),
$$

where $\Omega$ is the modified frequency

$$
\Omega=\omega \sqrt{\left(1-M_{c} \cos \theta\right)^{2}+\left(\alpha k^{1 / 2} / a_{0}\right)^{2}},
$$

where $\alpha$ is an experimental parameter with value of $0.5{ }^{12} \quad 198$

The length-scale $L$ can be calculated using parameters 199 obtained from a RANS $k-\varepsilon$ simulation as ${ }^{12,28}$

$$
L=c_{\ell} \frac{k^{3 / 2}}{\varepsilon},
$$

where $c_{\ell}$ is an empirical constant and $\varepsilon$ is the turbulent dissi- 201 pation rate. The time scale $\tau_{0}$ takes the form

$$
\tau_{0}=c_{\tau} \frac{k}{\varepsilon}
$$

where $c_{\tau}$ is an empirical constant.

Rewriting the length-scale in terms of the time scale Eq. 204 (12) takes the form

$$
I(\Omega)=\frac{\sqrt{\pi}}{4} \frac{c_{\ell}^{3}}{c_{\tau}^{3}} k^{7 / 2} \rho^{2} \tau_{0}^{4} \Omega^{4} \exp \left(-\frac{\tau_{0}^{2} \Omega^{2}}{8}\right),
$$

which gives the spectrum of the source emitting from a sin- 206 gle correlated volume of turbulence in the jet. Note that the 207 coefficient $c_{\tau}$ is in the definition of the time scale $\tau_{0}$; so even 208 if the term $c_{\ell}^{3} / c_{\tau}^{3}$ were combined as a single coefficient, $c_{\tau} 209$ would still be needed for $\tau_{0}$.

In Refs. $18-20$ a new time scale was proposed, which is 211 shown to better describe the energy transfer process related 212 to the jet noise generation process. The new time scale is 213 given by

$$
\tau_{0}^{\star}=\tau_{0}\left(\frac{L}{D}\right)^{2 / 3}
$$

where $D$ is the nozzle diameter. Replacing $\tau_{0}$ with $\tau_{0}^{\star}$ in Eq. 215 (16) and inserting the result in Eq. (1) yields

$$
\begin{aligned}
P(\boldsymbol{x} ; \omega)= & \frac{1}{64 \pi^{3 / 2}} \frac{1}{r^{2} a_{0}^{4}} \frac{c_{\ell}^{3}}{c_{\tau}^{3}} \int \Phi(\boldsymbol{x} \mid \boldsymbol{y}) D_{f}^{-5} \bar{\rho}^{2} k^{7 / 2} \\
& \times \tau_{0}^{\star 4} \Omega^{4} \exp \left(-\frac{\Omega^{2} \tau_{0}^{\star 2}}{8}\right) \mathrm{d}^{3} \boldsymbol{y} .
\end{aligned}
$$

In Sec. II B the ray tracing solution of the sound propa- 217 gation through the jet flow is presented and the associated 218 flow factor, $\Phi$, is introduced. 


\section{B. Propagation model}

A major drawback of LAA is that the refraction of sound by the mean flow is difficult to be accounted for because of the assumptions needed to describe the source term. Therefore alternative methods, for instance, through the definitions of the "Flow Factor" using the asymptotic solution of Lilley's equation, are necessary to model the effect of the mean flow. In this paper, we tackle this problem by introducing a Flow Factor parameter to take into account the sound-flow refraction phenomenon using a highfrequency approximation of sound propagation in nonuniform media by geometrical acoustics. The derivation of the ray tracing equations presented in this section follows the description of Pierce. ${ }^{30}$ The obvious advantage of the proposed technique to Lilley's asymptotic solution is its versatility and the possibility of using the new method for complex and asymmetric jet flows.

If $x_{p}^{\text {ray }}$ is a point on the wavefront defining the position of a ray, this point will follow the wavefront with velocity

$$
\frac{d \boldsymbol{x}_{\boldsymbol{p}}^{\mathrm{ray}}}{d t}=\boldsymbol{v}\left(\boldsymbol{x}_{\boldsymbol{p}}^{\mathrm{ray}}, t\right)+\boldsymbol{n}\left(\boldsymbol{x}_{p}^{\mathrm{ray}}, t\right) a\left(\boldsymbol{x}_{p}^{\mathrm{ray}}, t\right),
$$

where $\boldsymbol{n}$ is the vector normal to the wavefront. It is possible to calculate the ray path by integrating Eq. (19) with respect to time if $\boldsymbol{v}, a$, and $\boldsymbol{n}$ are known. However, the evaluation of $\boldsymbol{n}$ requires the reconstruction of the wavefront at each space time interval, which is not straightforward as it requires the position of all neighboring rays. A simpler solution is possible by using the wave-slowness vector, which is also normal to the wavefront and is defined as

$$
s=\frac{n}{a+v \cdot n},
$$

which can be written in the following form after some mathematical manipulation:

$$
s^{2}=\frac{\Omega^{2}}{a^{2}},
$$

where $\Omega=1-\boldsymbol{v} \cdot \boldsymbol{s}$. Equation (21) accounts for the slowness factor variation in space with the mean velocity and sound speed field.

The ray-tracing equations can be written in the Cartesian coordinate system, ${ }^{30}$ which are represented by six ordinary differential equations that couple the ray position and the slowness vector

$$
\begin{aligned}
& \frac{d x_{i}^{\text {ray }}}{d t}=U_{i}+\frac{a s_{i}}{1-U_{j} s_{j}}, \\
& \frac{d s_{i}}{d t}=-\frac{1-U_{j} s_{j}}{a} \frac{\partial a}{\partial x_{i}}-s_{j} \frac{U_{j}}{x_{i}} .
\end{aligned}
$$

The above system is solved by integrating Eqs. (22) and (23) in time using a fourth-order Runge-Kutta method, while the mean flow properties, i.e., $U_{i}$ and $a$ and associated derivatives, are obtained by interpolation from a numerical RANS flow-field solution. The equations are integrated until the ray exits the RANS simulation domain (i.e., unidirectional 261 flow), from where it is considered to follow a straight line to 262 the far-field observer position.

263

The ray tracing equations give no direct information 264 about the acoustic pressure amplitude. It is therefore neces- 265 sary to resort to the concept of ray-tubes and conservation of 266 energy which leads to the Blokhintzev invariant. ${ }^{30,31}$ The 267 invariant shows that along a given ray

$$
\frac{\overline{p^{2}} V A}{\left(1-U_{i} s_{i}\right) \rho a^{2}}=\text { const }
$$

where $p$ is the acoustic pressure, $V=\left|d \boldsymbol{x}^{\text {ray }} / d t\right|$ is the magni- 269 tude of the ray velocity vector, and $A$ is the ray-tube area. 270 Using Eq. (24) for a ray traced from the source location, $\boldsymbol{y}, 271$ to the far-field observer, $\boldsymbol{x}$, results in

$$
\frac{\left.\overline{p^{2}}\right|_{x}}{\left.\overline{p^{2}}\right|_{y}}=\frac{\left.\left.\frac{V}{\left(1-U_{i} s_{i}\right) \rho a^{2}}\right|_{y} A\right|_{y}}{\left.\frac{V}{\left(1-U_{i} s_{i}\right) \rho a^{2}}\right|_{x}} \frac{\left.A\right|_{x}}{x}
$$

which quantifies the change in the pressure amplitude along 273 a given ray from the source location to the far-field observer. 274 However, this is not the amplitude change needed to com- 275 pute the flow factor $\Phi$. The aim is to calculate the difference 276 of pressure amplitude in the far-field between a ray traced 277 over a quiescent medium and traced over the jet mean flow, 278 both launched from the same source location. Hence, the 279 flow factor used in our methodology is defined as

$$
\Phi(\boldsymbol{x}, \boldsymbol{y})=\frac{\left.\overline{p^{2}}\right|_{\boldsymbol{x}, \text { flow }}}{\left.\overline{\bar{p}^{2}}\right|_{\boldsymbol{x} \text {,quiescent }}},
$$

where $\left.\overline{p^{2}}\right|_{x \text { flow }}$ is evaluated at the observer location for a ray 281 launched from $\boldsymbol{y}$ and traced over the mean flow and 282 $\left.\overline{p^{2}}\right|_{x, \text { quiescent }}$ is evaluated at the observer location with the ray 283 traced over a quiescent medium (i.e., a straight line between 284 source and observer).

To compute $\Phi$ from Eq. (25) it is assumed that

$$
\begin{aligned}
& \left.\overline{p^{2}}\right|_{\boldsymbol{y}, \text { flow }}=\left.\overline{p^{2}}\right|_{\boldsymbol{y}, \text { quiescent }}, \\
& \left.\frac{V}{\left(1-U_{i} s_{i}\right) \rho a^{2}}\right|_{\boldsymbol{x}, \text { quiescent }}=\left.\frac{V}{\left(1-U_{i} s_{i}\right) \rho a^{2}}\right|_{\boldsymbol{y}, \text { quiescent }}
\end{aligned}
$$

and

$$
\left.A\right|_{y, \text { flow }}=\left.A\right|_{y, \text { quiescent }} .
$$

The flow factor can therefore be given by

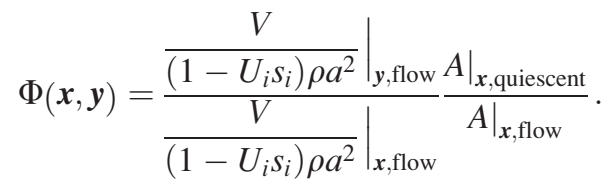

The first fraction on the right-hand side of Eq. (30) is 289 evaluated using the ray tracing solution and the flow infor- 290 mation obtained from the RANS solution. The ray-tube area 291 


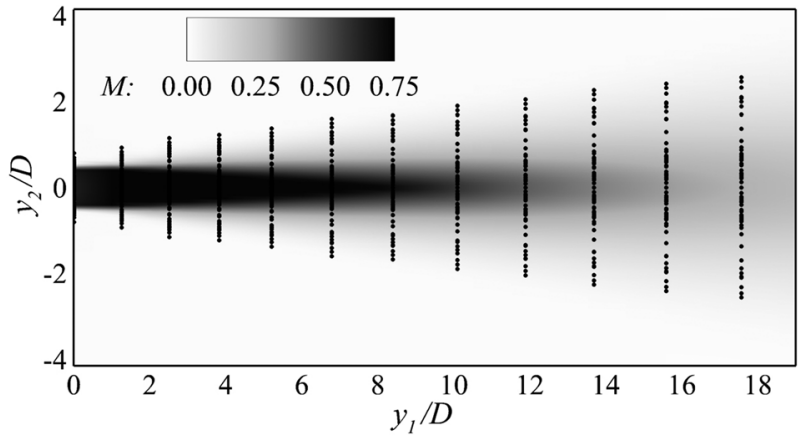

292

ratio cannot be computed directly from the ray tracing solution and is approximated by the ray density ratio in the far field.

To compute the ray density ratio, the far-field is represented as a spherical shell, discretized in spatial elements ( $\sim 10^{4}$ far-field bins for the results in this paper), and a large number of rays $\left(\sim 6 \times 10^{5}\right)$ are launched from each source location within the jet flow. To achieve a uniform spatial distribution, the far-field bins and the ray launching angles are defined using the vertices of a geodesic sphere. ${ }^{32-34}$ Each ray is assigned to a far-field bin by comparing its far-field location with the far-field bin coordinates. The number of rays assigned to each far-field bin is summed as $N_{\text {flow }}$ for rays traced through the mean flow and $N_{\text {quiescent }}$ when a quiescent medium is considered. Thus, Eq. (31) can be written as

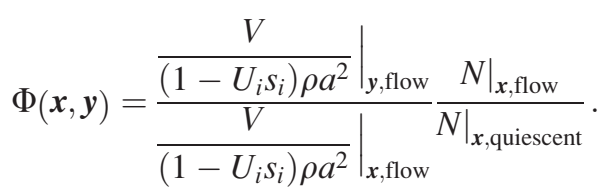

The flow factor $(\Phi)$ must now be calculated for a finite number of source locations $\boldsymbol{y}\left(\sim 10^{3}\right)$ within the jet domain. The locations are non-uniformly distributed in the jet domain, with clusters of sources in regions of high velocity gradients and turbulent kinetic energy. An example of the distribution of about 1700 sources for a single-flow jet is presented in Fig. 2. Having presented the source and propagation models, in Sec. III results for single-stream jets at different operating conditions will be presented and discussed.

\section{RESULTS AND COMPARISONS}

The canonical circular single-stream jet has been extensively studied analytically, numerically, and experimentally. ${ }^{15,26,29}$ In this section, some aspects of the sound

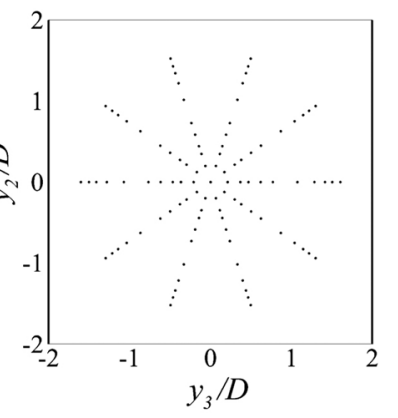

FIG. 2. Black dots show source locations for ray tracing method. generation of a circular single-stream jet at different oper- 320 ating conditions are presented and discussed using the 321 method developed in Sec. II. A total number of 12 operat- 322 ing conditions have been considered. They comprise three 323 Mach numbers: $M=0.5,0.75$, and 1.0 (reference sound 324 speed in the far-field is $340 \mathrm{~m} / \mathrm{s}$ ); and four temperature 325 ratios: $\mathrm{TR}=1.0,1.5,2.0$ and 2.5 (where TR is the ratio 326 between the jet-exit temperature and the reference temper- 327 ature of $288 \mathrm{~K}$ in the surrounding medium). The nozzle in 328 this study is shown in Fig. 3.

For each of the 12 cases, measurements of far-field spec- 330 tra are available and a corresponding CFD (Computational 331 Fluid Dynamics) RANS $k-\varepsilon$ solution is conducted. The 332 measurements of far-field noise were carried out in the Noise 333 Test Facility at QinetiQ Pyestock, United Kingdom. The 334 facility comprises of a chamber of area $27 \times 26 \mathrm{~m}^{2}$ and $14 \mathrm{~m} 335$ height, being anechoic down to approximately $90 \mathrm{~Hz}$. Results 336 used in this paper are recorded using a microphone array at 337 $12 \mathrm{~m}(\approx 120 \mathrm{D})$ from the nozzle exit and are presented as $1 \mathrm{~m} 338$ loss-less data.

A brief description of the mean flow solution is pre- 340 sented in Sec. III A, followed by a presentation of the results 341 computed with the source and propagation models presented 342 in this paper. The main emphasis of the results is to show the 343 accuracy in the far-field noise prediction and the possibility 344 to account for three-dimensional propagation effects for a 345 realistic spreading jet.

\section{A. Mean flow solution}

The mean flow is computed with a standard finite volume 348 second-order commercial CFD solver. ${ }^{35}$ The continuity, 349 momentum, and energy equations are solved for a compressible 350 gas, along with the equation of state for an ideal gas. To model 351 the jet flow the standard $k-\varepsilon$ model is used, with the two addi- 352 tional equations solved using the standard coefficients.
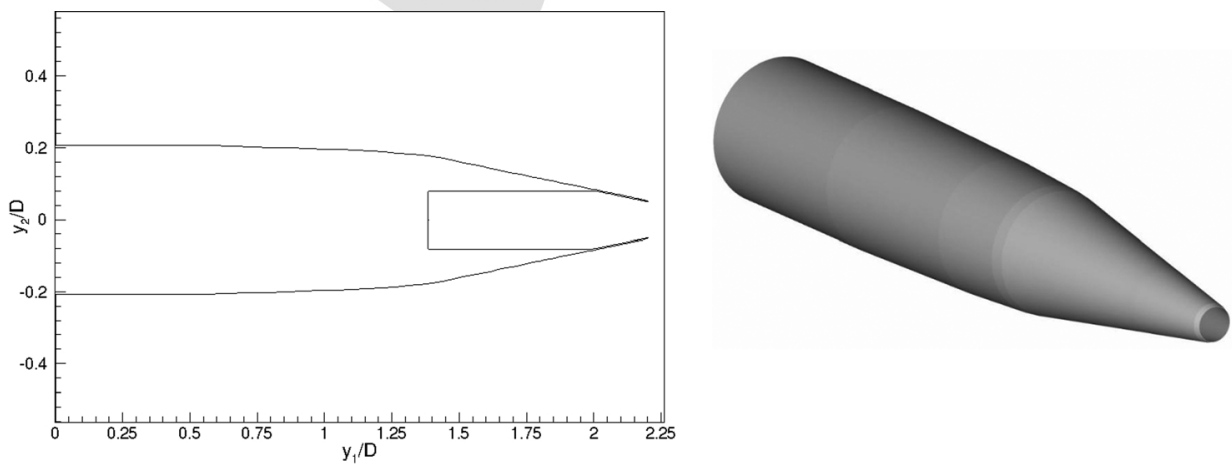

FIG. 3. Geometry of the $D=0.1016 \mathrm{~m}$ nozzle. 


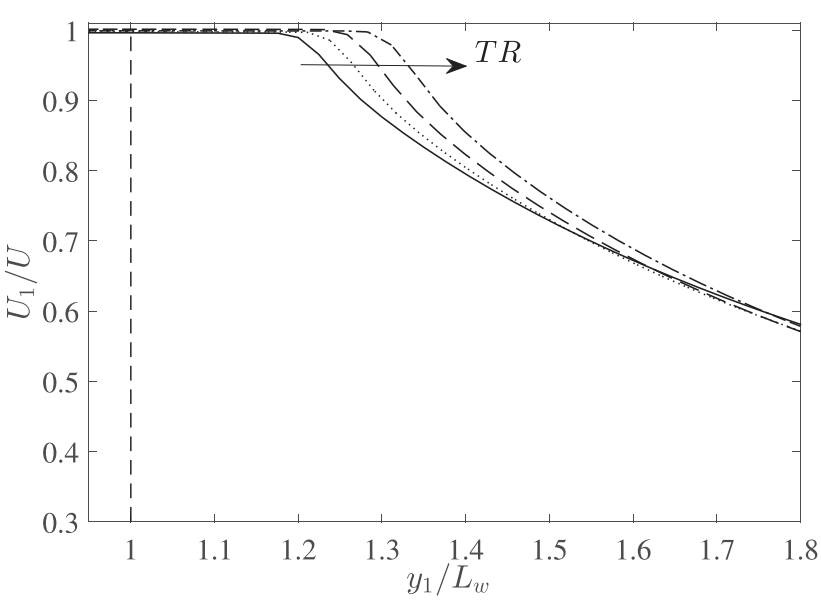

FIG. 4. Centerline axial velocity decay with axial distance normalized by empirical length of potential core $\left(L_{w}\right)$ (Ref. 37) for $M=0.75$ jets. Solid line, $\mathrm{TR}=1$; dotted line, $\mathrm{TR}=1.5$; dashed line $\mathrm{TR}=2$; and dashed-dotted line $\mathrm{TR}=2.5$. The parameter $L_{w}$ was computed for each temperature ratio. The fact that the curves start to decay at higher $y_{1} / L_{w}$ shows that the overprediction of the potential core length by RANS $k-\varepsilon$ worsens with increased temperature ratio.

Figure 4 shows the normalized velocity along the jet center-line for a $M=0.75$ jet at different temperature ratios, $\mathrm{TR}=1,1.5,2$, and 2.5. Results are presented in terms of the empirical potential core length as defined by $\mathrm{Witze}^{37}$

$$
L_{w}=(D / 2)\left[0.08(1-0.16 M) \mathrm{TR}^{0.28}\right]^{-1},
$$

so that $y_{1} / L_{w}=1$ represents the end of potential core for a given $M$ and TR. As known, the predictions with the standard $k-\varepsilon$ model result in an over-prediction of the potential core length. Although several turbulence model corrections have been proposed and discussed in the literature, ${ }^{36}$ we have used the standard model as it is widely available and used in an industrial context. As can be seen, the overprediction grows with the temperature ratio (TR), making the predictions less reliable for very hot jets. Despite the obvious shortcomings of the $k-\varepsilon$ model, the mean flow solution is still capable of providing good jet noise prediction, which will be discussed in Secs. III B-III E.

\section{B. Far-field noise prediction at $90^{\circ}$}

RANS-based prediction methods ${ }^{14-16,38,39}$ generally require empirically calibrated coefficients to relate the statistical properties of the mean flow from RANS $k-\varepsilon$ to the relevant properties of the sound generation process (or, more recently, calibrated with transient numerical solutions). ${ }^{40,41}$ Contrary to other methods that rely on three coefficients (amplitude, length-scale, and time scale), the method presented in this paper only needs two coefficients, $c_{\ell}$ and $c_{\tau}$. The values for these coefficients are computed by comparing the predicted SPL with the measured noise data at $\theta=90^{\circ}$. The optimum values vary slightly with Mach number but more significantly with temperature ratio. The jet noise predictions for isothermal jets are performed using $c_{\tau}=0.43$ and $c_{\ell}=0.8$. For hot jets $c_{\tau}$ is kept at the same value while $c_{\ell}$ is allowed to vary from 0.8 for $\mathrm{TR}=1$ to around 1.9 for $\mathrm{TR}=2.5$.
Figure 5 shows a comparison of the predicted SPL at 387 $\theta=90^{\circ}$ with measured far-field data for the 12 cases consid- 388 ered, in the absence of refraction effects. The good agree- 389 ment observed, both in terms of the overall shape of the 390 spectra and the peak frequency location at different Mach 391 numbers, confirms that the source model captures well the 392 physics of the noise generation mechanism. The need of cali- 393 bration for different temperature ratios is a result of neglect- 394 ing the additional source terms related to hot jets, such as the 395 density variation. Nevertheless, by showing that $c_{\tau}$ can be 396 kept constant while only $c_{\ell}$ needs further calibration to prop- 397 erly capture the SPL spectra of the hot jets is an indication 398 that this additional source has a similar nature of the source 399 already modeled.

\section{Source location results}

The source model developed in Sec. II can be used to 402 study the distribution of the sound sources in the jet plume. 403 To do so, the volume integral in Eq. (18) is computed only 404 in the $y_{2}-y_{3}$ plane so the contribution to the far-field noise 405 from a slice of the jet is computed as $P_{\text {slice }}\left(\boldsymbol{x}, \omega, y_{1}\right)$. $\quad 406$

Figure 6 shows the results for an observer located at $90^{\circ} 407$ in the far-field. Different Strouhal numbers $(\mathrm{St}=f D / U)$ for 408 isothermal jets at Mach numbers of 0.5, 0.75, and 1 are con- 409 sidered. The source amplitude results are normalized by its 410 value at $\mathrm{St}=0.2$. As expected, results have shown the 411 higher-frequency sources are located near the nozzle exit 412 and the most energetic sources are slightly after the end 413 potential core (if the overprediction of the potential core 414 length shown in Fig. 4 is considered, the peak in Fig. 6415 moves closer to the end of potential core). The collapsing of 416 the results for the three different Mach numbers is evidence 417 that the source distribution is self-similar in frequency and 418 space, with the driving parameters being the Strouhal num- 419 ber for frequency and $y_{1} / L_{w}$ for space.

\section{Sound-flow interaction effects}

The effect of refraction can further be analyzed in isola- 422 tion by plotting the flow factor computed using the ray trac- 423 ing and ray density ratio. The flow factor $\Phi(\boldsymbol{x} \mid \boldsymbol{y})$ gives the 424 amplification or reduction of the SPL due to the refraction 425 for the noise collected at a microphone location $(\boldsymbol{x})$ due to a 426 noise source at $(\boldsymbol{y})$ within the jet plume. In this section, the 427 flow factor results in $\mathrm{dB}$, i.e., $(10 \log \Phi)$, are presented in 428 two forms: (i) by fixing the source location $(\boldsymbol{y})$ and varying 429 the observer location $(\boldsymbol{x})$ in the far-field over $0^{\circ}<\theta<180^{\circ} 430$ and $0^{\circ}<\phi<360^{\circ}$, and (ii) fixing the observer location (x) 431 and varying the source location $\left(y_{1}\right.$ and $\left.y_{2}\right)$ within the jet 432 plume. This enables a better understanding of the three- 433 dimensional nature of the refraction effects appearing even 434 in the axisymmetry nozzle studied in this paper. 435

First, the effect of refraction is analyzed for sound emit- 436 ted from sources on the lip-line of a $M=0.75$ jet with 437 $\mathrm{TR}=1$, see Fig. 7. The sources are positioned along the noz- 438 zle lip-line $\left(y_{2} / D=0.5\right)$, i.e., within the jet shear-layer 439 where the turbulent kinetic energy $(k)$ peaks and, according 440 to $P(\omega) \propto k^{7 / 2}$ relation, from Eq. (18), can be considered as 441 one of the most important noise generation regions. Figure 7442 

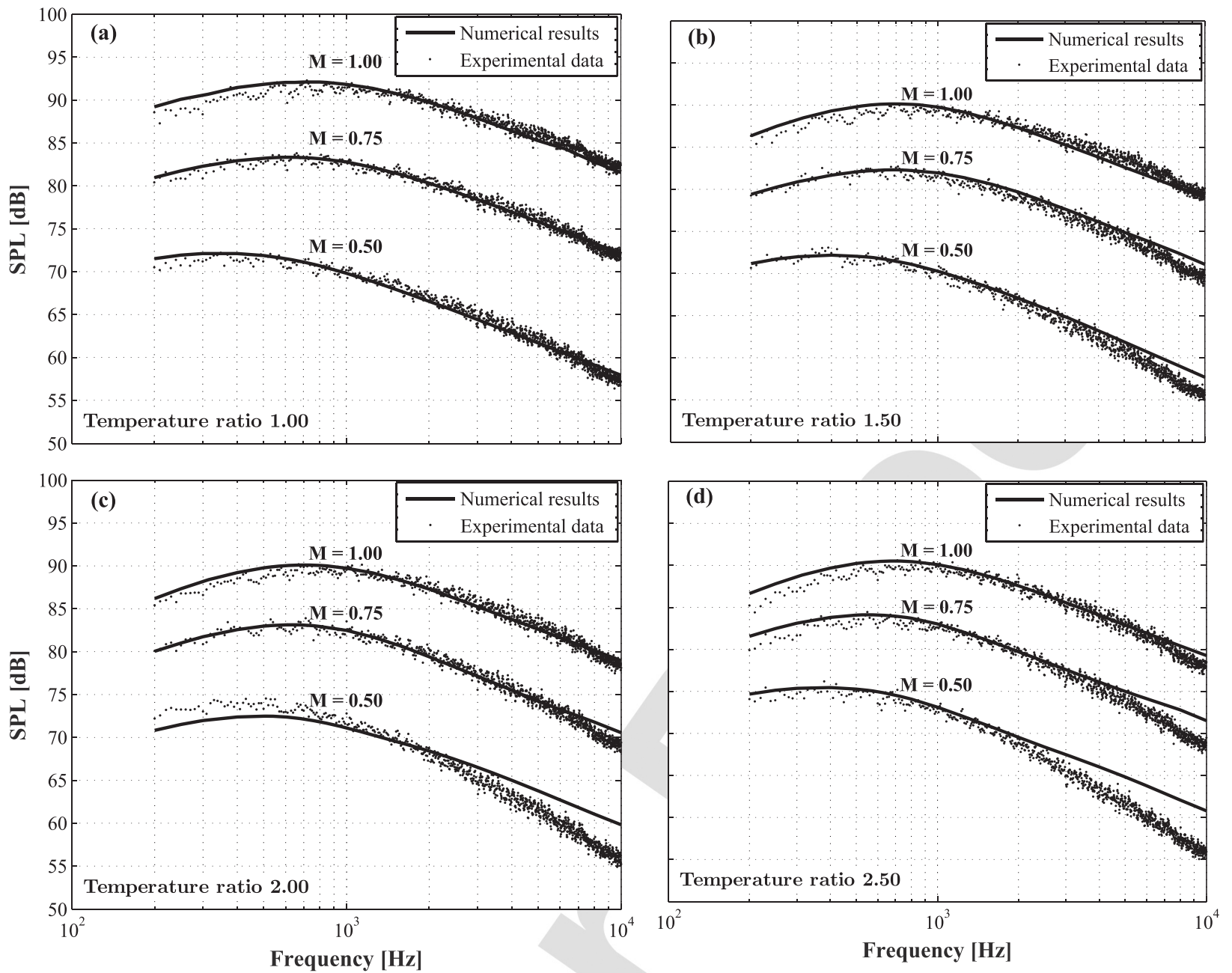

FIG. 5. Far-field SPL predictions and measurements at $90^{\circ}$ for different $M$ and TR: (a) TR $=1.00,(b) \mathrm{TR}=1.50,(\mathrm{c}) \mathrm{TR}=2.00$, (d) $\mathrm{TR}=2.50$.

shows the contour plots of the flow factor, where the negative Flow Factor indicates reduction of SPL due to the flow refraction and positive values show sound amplification. The white area in the plots represents the shadow zone where no rays are collected and the ray tracing approximation is no longer valid. The effects of refraction are presented as a function of the polar and azimuthal angles of the observer for sound emitted from four different source locations on the lip line with different downstream locations $\left(y_{1} / D=1,2.6\right.$, 5 , and 10).

For a source located at $y_{1} / D=1$ and $y_{2} / D=0.5$, the shadow zone has a variable shape along the azimuthal coordinate, see Fig. 7(a). The dashed line $A$ shows that the critical angle defining the shadow zone occurs at about $60^{\circ}$ and it goes from $\varphi \approx 10^{\circ}$ to $160^{\circ}$. With increasing $\varphi$, a new shadow zone area will appear, shown as region $B$. The change of the critical angle down to $\theta=20^{\circ}$ for observers in the opposite side of the source is an interesting phenomenon which has not previously been shown. An area of high intensity, i.e., sound amplification, can also be observed within region $\mathrm{B}$, at about $\theta=65^{\circ}$, which is due to the rays entering the potential core of the jet, i.e., the rays that are not being totally reflected. The potential core in this situation acts like a lens for these rays, focusing them over a small region. This shows the importance of the effect of the potential core on sound propagation within the jet plume and the far-field 468 noise amplification, particularly for asymmetric jets. 469 Another area of strong sound amplification for observers 470 below the jet occurs at $\varphi \approx 90^{\circ}$ and $\theta \approx 110^{\circ}$, shown as 471 Region C.



FIG. 6. Source distribution for isothermal jets as a function of axial distance for different Strouhal number $(\mathrm{St}=f D / U)$, normalized by the maximum of the distribution for $\mathrm{St}=0.2$. Axial coordinate normalized by potential core length $\left(L_{w}\right)$. Solid lines, $M=0.5$; dashed lines, $M=0.75$; dotted lines, $M=1$. 

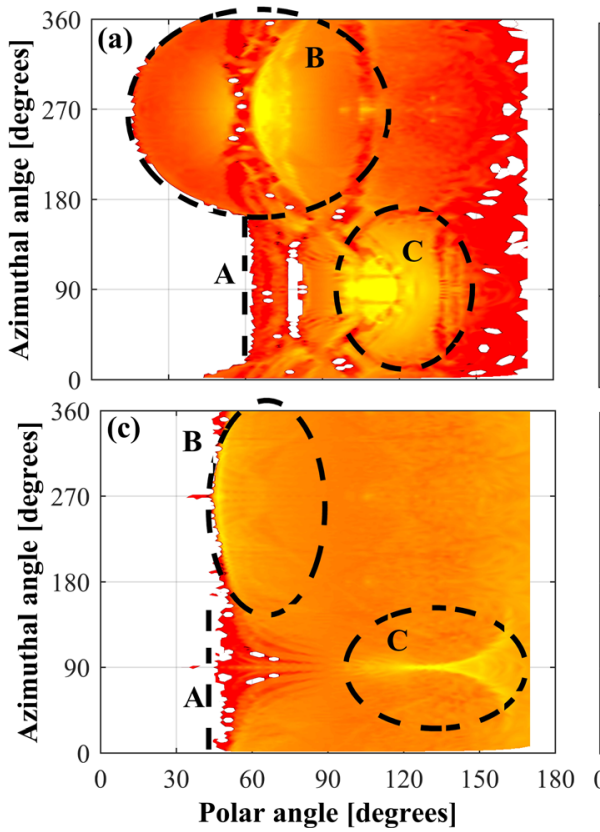

Moving further downstream, for a point source located at $y_{1} / D=2.6$ and $y_{2} / D=0.5$, Fig. $7(\mathrm{~b})$, the Flow Factor results change considerably, altering not only its shape but also the critical angle to $\approx 40^{\circ}$. Also, the noise amplification region before the shadow zone still plays an important role for this source location. Regarding region $\mathrm{C}$, the peak area is becoming sharper and it is spreading along the polar angles. This can be understood by the fact that more rays are being convected by the flow due to the jet spreading. A similar trend has been observed for a source located near the end of the potential core at $y_{1} / D=5$ and $y_{2} / D=0.5$, see Fig. 9(c). The main differences are that the critical angle (shown by line A) goes down to $\approx 45^{\circ}$ and varies less with $\varphi$. Since the point source is now located near the end of the potential core, the acoustic lens effect of the potential core, as observed in Fig. 7(a) (region B), become less obvious and
Region B shrink to a very small $\theta$ area over 489 $180^{\circ}<\phi<360^{\circ}$. Region $\mathrm{C}$ also moves to higher polar 490 angles of about $\theta=140^{\circ}$. The results in Fig. 7(d) show that 491 in the case of a source positioned at $y_{1} / D=10$ and 492 $y_{2} / D=0.5$, in the absence of strong velocity gradient, the 493 blockage effect (for $\varphi \approx 270^{\circ}$ ) is minimized and it is no lon- 494 ger possible to identify regions B and C. Following the trend 495 from the previous source locations, the critical angle shown 496 by line $\mathrm{A}$ is further reduced to $\theta \approx 20^{\circ}$ and becomes effec- 497 tively axisymmetric.

The results in Figs. 8 and 9 show the flow factor for dif- 499 ferent regions of the jet for an observer at $\varphi=90^{\circ}$ (i.e., 500 above the plane of the figure) and two different polar angles 501 $\left(\theta=50^{\circ}\right.$ and $\left.\theta=90^{\circ}\right)$. Results are presented for an isother- 502 mal and $\mathrm{TR}=2.5$ jet. As expected, the refraction factor in 503 the case of an observer at $\theta=90^{\circ}$ is almost zero, indicating 504
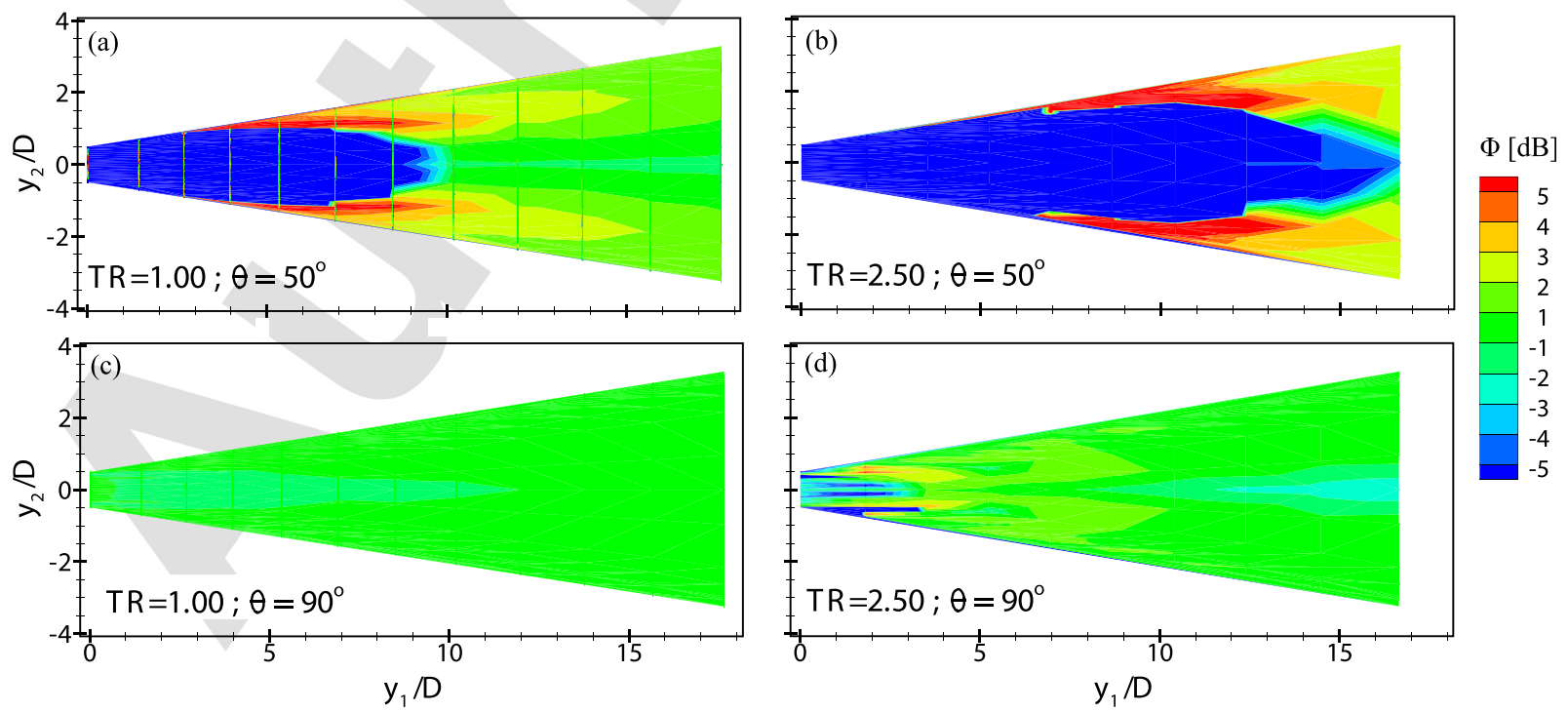

FIG. 8. (Color online) Flow factor for jet with $M=0.75$ and different temperature ratios: (a) and (c), TR $=1$; (b) and (d), TR $=2.5$. Observer above plane of figure $\left(\varphi=90^{\circ}\right)$ and different polar angles: (a) and (b), $\theta=50^{\circ}$; (c) and (d), $\theta=90^{\circ}$. 

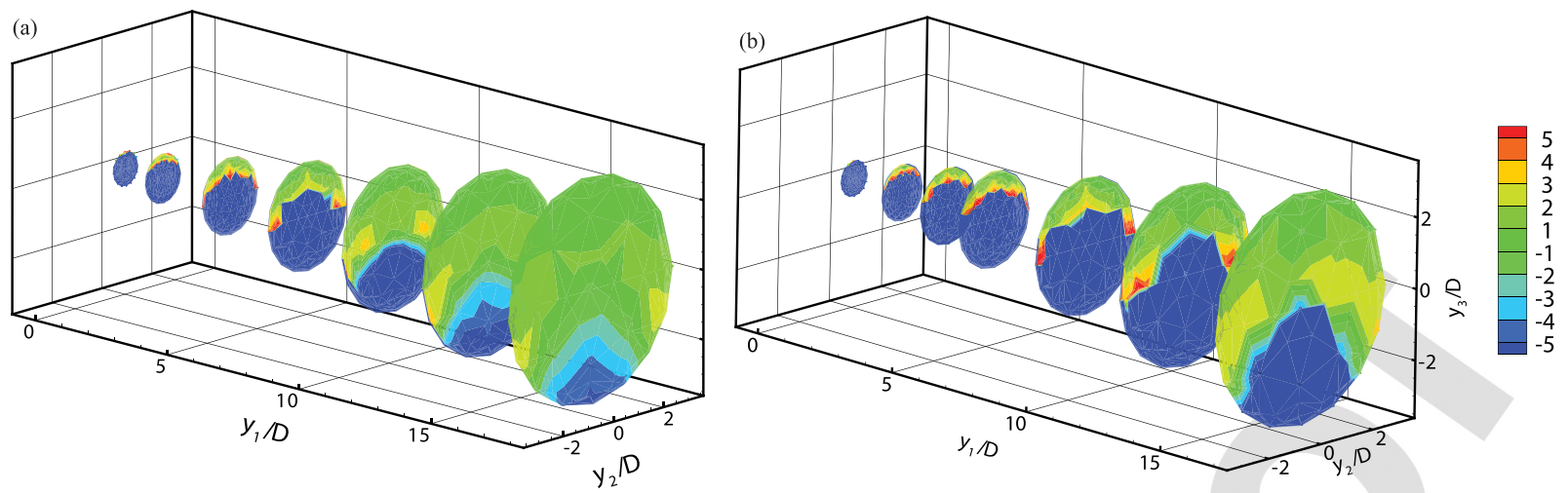

FIG. 9. (Color online) Three-dimensional visualization of flow factor for $M=0.75$ with different temperature ratios: (a) $\mathrm{TR}=1$, (b) TR $=2.5$. Far-field observer at $\theta=50^{\circ}$ and $\varphi=0^{\circ}$.

very small refraction effects due to the sound and flow interactions. At small polar angles, Figs. 8(a) and 8(b), however, the regions close to the nozzle, where the velocity gradient is large, is significantly affected. Increasing the temperature ratio has also been shown to increase the level of refraction effects. The flow factor results over the $y_{1}-y_{2}$ planes at different axial locations for an observer located at $\varphi=90^{\circ}$ and $\theta=50^{\circ}$ are presented in Fig. 9. The results clearly show that the refraction due to the sound-flow interaction in an axisymmetric jet flow is not axisymmetric and the sources located on the opposite side of the observer suffer more refraction effects. As observed in Fig. 8, increasing the jet temperature ratio increases the region of the jet affected by refraction, Fig. 9(b).

\section{E. Far-field noise directivity}

To assess the ray-tracing based propagation model developed here, the far-field SPL results at different polar angles are presented for different Mach numbers, $M=0.5$, 0.75 , and 1.00 , at $\mathrm{TR}=1$, see Fig. 10. Results are presented for observers outside the zone of silence at $\theta=60^{\circ}$ and $110^{\circ}$ from the jet axis. Results show that the far-field noise can be 525 generally captured well for observers outside the zone of 526 silence using the source and refraction model. The issue of 527 propagation into the zone of silence and the limitations of 528 the method will be discussed later.

Having shown that both the spectral behavior of the far- 530 field noise at $90^{\circ}$ (Fig. 5) and at different polar angles (Fig. 531 10), and also the Flow Factor at different jet operating condi- 532 tions (Figs. 7-9), we shall now study the overall sound pres- 533 sure level (OASPL) for polar angles in the range of 534 $30^{\circ}-120^{\circ}$, see Figs. 11 and 12. Figure 11 shows the OASPL 535 results for jets at $M=0.5$ and 0.75 at different temperature 536 ratios ( $\mathrm{TR}=1.0,1.5,2$, and 2.5). Results for a $M=0.5$ jet 537 show that the critical angle in the case of $\mathrm{TR}=1$ occurs at 538 about $46^{\circ}$ and it moves to higher angles with temperature 539 ratio. As expected, the model fails to predict the far-field 540 noise within the zone of silence, but provides very good 541 agreement at angles greater than the critical angle. The far- 542 field noise comparisons for a $M=0.75$ jet also show that the 543 model developed in this work is capable of predicting the 544 OASPL very accurately outside the zone of silence. It can 545 also be seen from the experimental data that the far-field 546
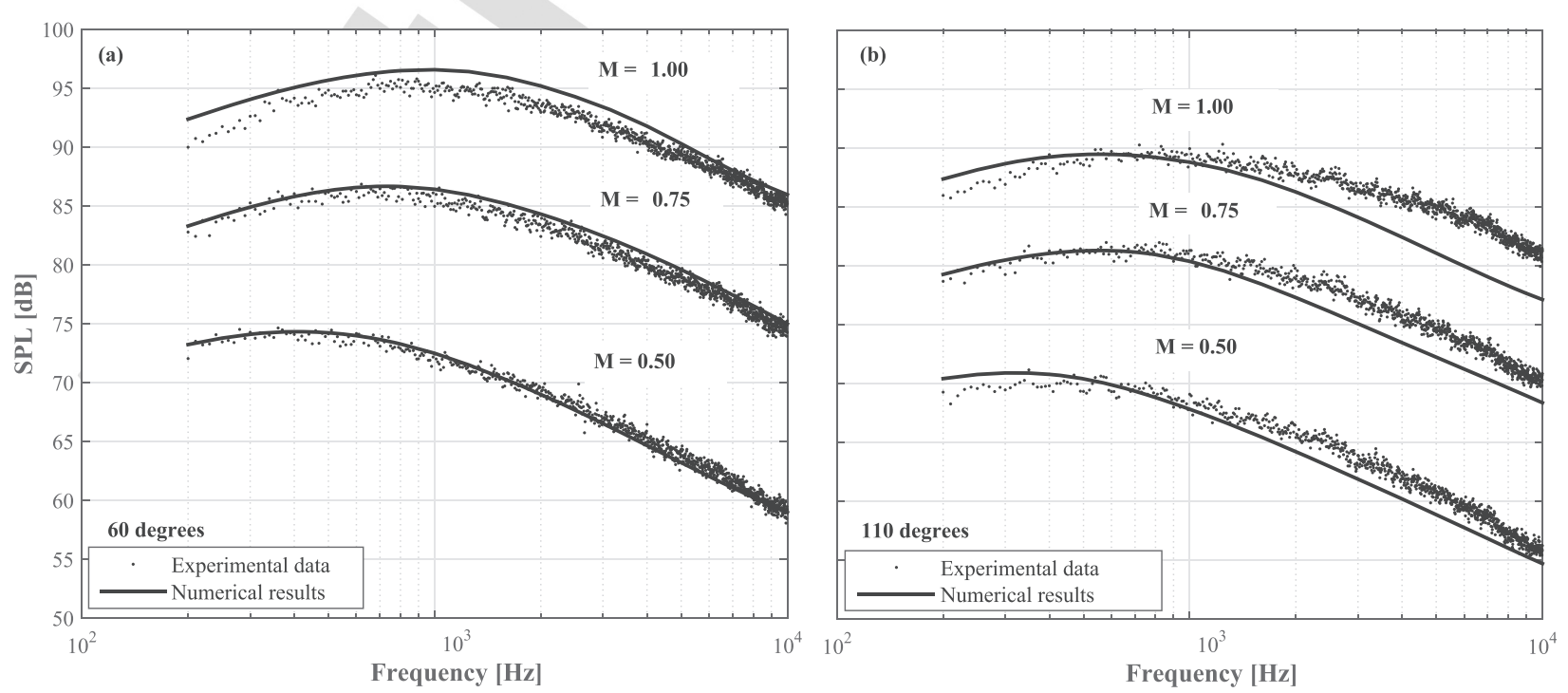

FIG. 10. Far-field SPL predictions and measurements at $60^{\circ}$ and $110^{\circ}$ for the isothermal jet with different $M$ : (a) $\theta=60^{\circ}$, (b) $\theta=110^{\circ}$. 

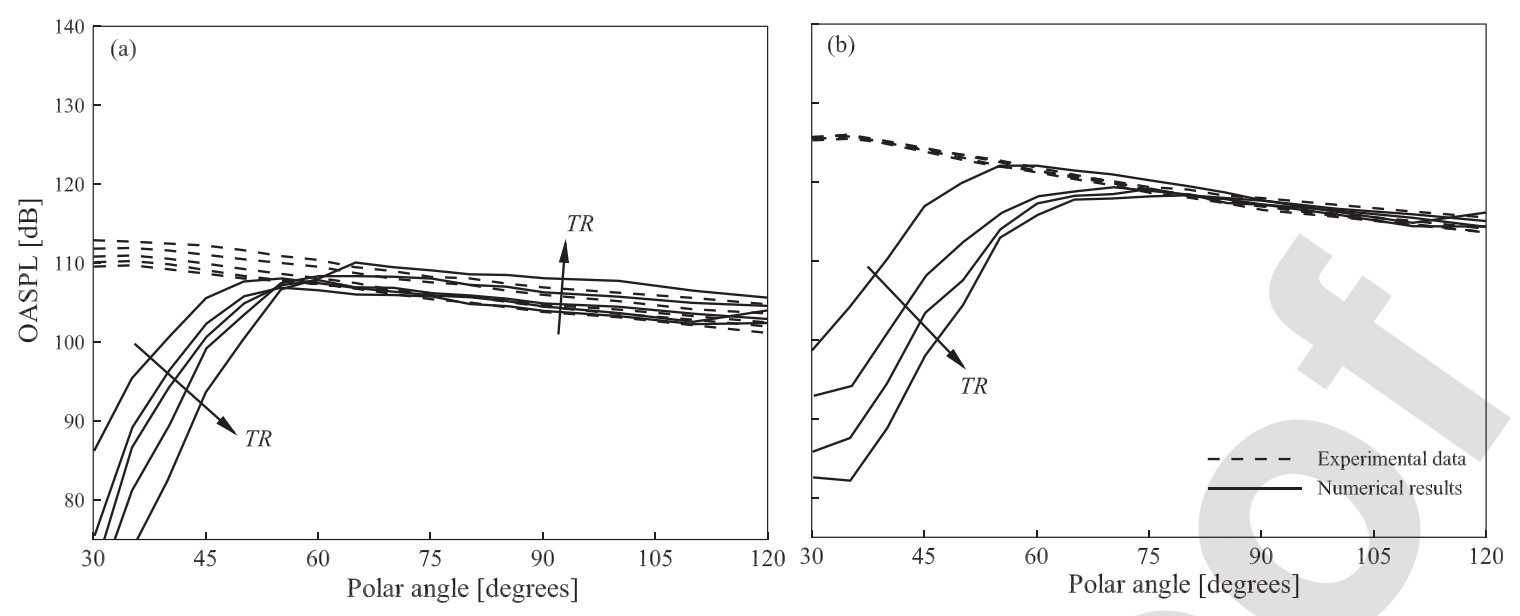

FIG. 11. OASPL prediction (solid lines) and measurements (dashed lines) for (a) $M=0.5$ and (b) $M=0.75$ with temperature ratio ranging from 1.0 to 2.5 .

noise is more sensitive to temperature ratio at low Mach numbers $(M=0.5)$, and that the source and propagation models have managed to predict this effect well.

\section{CONCLUSIONS}

In this paper an application of the LAA to model the sources of jet mixing noise coupled to a ray tracing method to compute effects of refraction is presented. The resulting method is a promising solution to quickly evaluate the noise emitted by jets from arbitrary nozzle geometries. This is particularly desired in an industrial context as it relies on the standard RANS $k-\varepsilon$ solution and makes no further assumption about the flow. Despite the need of calibration with farfield measurements, only two coefficients are needed instead of three as it is usually the case for similar methods from the literature. The coefficients are fixed for isothermal jets in the subsonic regime, however one of them needs to be changed with increasing temperature ratio; such need is understood to result from the neglect of the enthalpy source arising in heated jets. $^{42-47}$

Results show that the method proposed in this paper captures well the contribution of fine-scale turbulence to jet mixing noise in the subsonic regime down to a polar angle

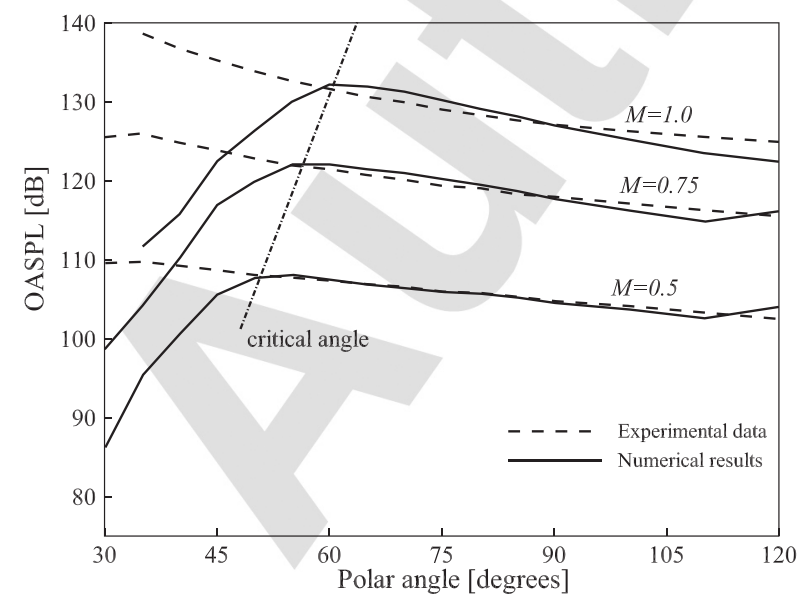

FIG. 12. OASPL prediction (solid lines) and measurements (dashed lines) at $\mathrm{TR}=1.0$ with Mach number ranging from 0.5 to 1.0 . The critical angle is shown to increase linearly with $M$. of $50^{\circ}$, below which the effect of a shadow zone invalidates 569 the real ray tracing assumption. Such range of observer 570 angles (above $50^{\circ}$ ) give valuable information if a quick esti- 571 mation of the impact of non-axisymmetric geometries is 572 sought. It thus satisfies the requirement of a design tool, pre- 573 senting reasonable accuracy at relatively low computational 574 cost while being able to consider general three-dimensional 575 nozzle geometries.

ACKNOWLEDGMENTS

The authors thank the financial support from CAPES, 578 FAPESP, and EMBRAER. This research has been 579 conducted using data from the SYMPHONY project, which 580 is part of the UK Technology Strategy Board Contract No. 581 TP11/HVM/6/I/AB201K.

${ }^{1}$ D. Papamoschou and M. Debiasi, "Directional suppression of noise from a 584 high-speed jet," AIAA J. 39(3), 380-387 (2001).

${ }^{2} \mathrm{~J}$. Bridges and C. A. Brown, "Parametric testing of chevrons on single 586 flow hot jets," in Proceedings of the 10th AIAA/CEAS Aeroacoustics 587 Conference, AIAA Paper No. 2004-2824 (2004). 588

${ }^{3}$ D. Papamoschou, "New method for jet noise reduction in turbofan 589 engines," AIAA J. 42(11), 2245-2253 (2004). 590

${ }^{4}$ K. Viswanathan, M. Shur, M. Strelets, and P. R. Spalart, "Numerical pre- 591 diction of noise from round and beveled nozzles," in Proceedings of the 592 EUROMECH Colloquium 467: Turbulent Flow and Noise Generation 593 (2005). $\quad 594$ ${ }^{5}$ ESDU International plc, "ESDU 98019 and software B9819: Computer- 595 based estimation procedure for single-stream jet noise" (1998). 596 ${ }^{6} \mathrm{~J}$. B. Freund, "Noise sources in a low-Reynolds-number turbulent jet at 597 Mach 0.9," J. Fluid Mech. 438, 277-305 (2001). 598

${ }^{7}$ C. Bogey, S. Barré, D. Juvé, and C. Bailly, "Simulation of a hot coaxial 599 jet: Direct noise prediction and flow-acoustics correlations," Phys. Fluids 600 21(3), 035105 (2009).

${ }^{8}$ H. Xia, P. G. Tucker, and S. Eastwood, "Large-eddy simulations of chev- 602 ron jet flows with noise predictions," Int. J. Heat Fluid Fl. 30(6), 603 1067-1079 (2009). 604

${ }^{9}$ T. F. Balsa and P. R. Gliebe, "Aerodynamics and noise of coaxial jets," 605 AIAA J. 15(11), 1550-1558 (1977). 606

${ }^{10}$ T. F. Balsa, P. R. Gliebe, R. A. Kantola, R. Mani, E. J. Stringas, and J. C. 607 F. Wang, "High velocity jet noise source location and reduction. Task 2: 608 Theoretical developments and basic experiments," Technical Report 609 FAA-RD-76-II, Federal Aviation Administration (1978). 610

${ }^{11}$ G. M. Lilley, "The generation and radiation of supersonic jet noise. Vol. 611 IV-Theory of turbulent generated jet noise, noise radiation from 612 upstream sources, and combustion noise. Part, I. I. Generation of sound in 613 a mixing region," Technical report, Air Force Propulsion Laboratory 614 (1972). 
$616{ }^{12}$ A. Khavaran, E. A. Krejsa, and C. M. Kim, "Computation of supersonic 617 jet mixing noise for an axisymmetric cd nozzle using k-epsilon turbulence 618 model," in Proceedings of the 30th Aerospace Sciences Meeting and 619 Exhibit, AIAA Paper No. 92-0500 (1992).

$620{ }^{13}$ A. Khavaran and E. A. Krejsa, "Role of anisotropy in turbulent mixing 621 noise," AIAA J. 37(2), 832-941 (1998).

$622{ }^{14} \mathrm{C}$. K. W. Tam and L. Auriault, "Jet mixing noise from fine-scale 623 turbulence," AIAA J. 37(2), 145-153 (1999).

$624{ }^{15} \mathrm{P}$. J. Morris and F. Farassat, "Acoustic analogy and alternative theories for 625 jet noise prediction,” AIAA J. 40(4), 671-680 (2002).

$626{ }^{16}$ R. H. Self, "Jet noise prediction using the Lighthill acoustic analogy," 627 J. Sound Vib. 275(3-5), 757-768 (2004).

$628{ }^{17}$ M. Harper-Bourne, "Jet near-field noise prediction," in Proceedings of the 5th AIAA/CEAS Aeroacoustics Conference and Exhibit, AIAA Paper No. 99-27825 (1999).

${ }^{18}$ R. H. Self and M. Azarpeyvand, "Utilization of turbulent energy transfer rate time-scale in aeroacoustics with application to heated jets," Int. J. Aeroacoust. 7(2), 83-102 (2008).

${ }^{19}$ R. H. Self and M. Azarpeyvand, "Jet noise prediction using different turbulent scales," Acoust. Phys. 55(3), 433-440 (2009).

${ }^{20} \mathrm{M}$. Azarpeyvand and R. H. Self, "Improved jet noise modeling using a new time-scale,” J. Acoust. Soc. Am. 126(3), 1015-1025 (2009).

${ }^{21}$ S. M. Candel, "Numerical solution of conservation equations arising in linear wave theory: Application to aeroacoustics," J. Fluid Mech. 83(3), 465-493 (1977).

${ }^{22}$ J. B. Freund and T. G. Fleischman, "Ray traces through unsteady jet turbulence," Int. J. Aeroacoust. 1(1), 83-96 (2002).

${ }^{23}$ P. R. Spalart, M. L. Shur, and M. Kh. Strelets, "Identification of sound sources in large-eddy simulations of jets," in Proceedings of the 13th AIAA/CEAS Aeroacoustics Conference, AIAA Paper No. 2007-3616 (2007).

${ }^{24}$ X. Xu, J. He, X. Li, and F. Q. Hu, “3-D jet noise prediction for separate flow nozzles with pylon interaction," in Proceedings of the 53rd AIAA Aerospace Sciences Meeting, AIAA Paper No. AIAA 2015-0512 (2015).

${ }^{25}$ M. J. Lighthill, "On sound generated aerodynamically. I. General theory," Proc. Roy. Soc. A 211, 564-587 (1952).

${ }^{26}$ H. S. Ribner, "Quadrupole correlations governing the pattern of jet noise," J. Fluid Mech. 38(1), 1-24 (1969).

${ }^{27} \mathrm{G}$. K. Batchelor, The Theory of Homogeneous Turbulence (Cambridge University Press, Cambridge, 1953).

${ }^{28} \mathrm{C}$. Bailly, W. Béchara, and P. Lafon, "Jet noise prediction using a kepsilon turbulence model," in Proceedings of the 15th AIAA Aeroacoustics Conference, AIAA Paper No. 93-4412 (1993).

${ }^{29}$ P. A. Lush, "Measurements of subsonic jet noise and comparison with theory," J. Fluid Mech. 46(3), 477-500 (1971).
${ }^{30}$ A. D. Pierce, Acoustics: An Introduction to its Physical Principles and 662 Applications (McGraw-Hill, New York, 1981). 663

${ }^{31} \mathrm{D}$. Blokhintzev, "The propagation of sound in an inhomogeneous and 664 moving medium I," J. Acoust. Soc. Am. 18(2), 322-328 (1946). 665

${ }^{32}$ H. Kenner, Geodesic Math and How to Use It (University of California 666 Press, Oakland, CA, 1976).

${ }^{33}$ M. J. Wenninger, Spherical Models (Cambridge University Press, 668 Cambridge, 1979). $\quad 669$

${ }^{34}$ S. Y. Seidel, "Site-specific propagation prediction for wireless in-building 670 personal communication system design," IEEE Trans. Vehicular Technol. 671 43(4), 879-891 (1994). 672

${ }^{35}$ ANSYS Inc., Canonsburg, PA, Product Documentation Release 14.0673 (2010). 674

${ }^{36}$ C. K. W. Tam and A. Ganesan, "Modified k-epsilon turbulence model for 675 calculating hot jet mean flows and noise," AIAA J. 42(1), 26-34 (2004). 676

${ }^{37}$ P. O. Witze, "Centerline velocity decay of compressible free jets," AIAA J. 677 12(4), 417-418 (1974). 678

${ }^{38} \mathrm{P}$. J. Morris and S. Boluriaan, "The prediction of jet noise from CFD 679 data," in Proceedings of the 10th AIAA/CEAS Aeroacoustics Conference, 680 AIAA Paper No. 2004-2977 (2004). 681

${ }^{39}$ M. Omais, S. Redonnet, B. Caruelle, and E. Manoha, "Jet noise prediction 682 using RANS CFD input," in Proceedings of the 5th AIAA/CEAS 683 Aeroacoustics Conference, AIAA Paper No. 2008-2938 (2008). 684

${ }^{40}$ S. A. Karabasov, M. Z. Afsar, T. P. Hynes, A. P. Dowling, W. A. 685 McMullan, C. D. Pokora, G. J. Page, and J. J. McGuirk, "Jet noise: 686 Acoustic analogy informed by Large Eddy Simulation," AIAA J. 48(7), 687 1312-1325 (2010).

${ }^{41}$ N. K. Depuru Mohan, A. P. Dowling, S. A. Karabasov, H. Xia, O. 689 Graham, T. P. Hynes, and P. G. Tucker, "Acoustic sources and far-field 690 noise of chevron and round jets,” AIAA J. 53(9), 2421-2436 (2015). 691

${ }^{42}$ M. E. Goldstein, Aeroacoustics (McGraw-Hill, New York, 1976). 692

${ }^{43}$ R. H. Self, "A RANS CFD coupled model for predicting coaxial jet 693 noise," ISVR Internal Technical report No. 304, Institute of Sound and 694 Vibration Research, Southampton, U. K. (2005). 695

${ }^{44}$ C. R. S. Ilário, "Development of a novel RANS-based method for the 696 computational aeroacoustics of high speed jets," Ph.D. thesis, 697 Universidade de São Paulo, São Paulo, 2011. 698

${ }^{45}$ R. C. Engel, C. R. S. Ilário, and C. J. Deschamps, "Application of RANS- 699 based method to predict acoustic noise of chevron nozzles," Appl. Acoust. 700 79, 153-163 (2014). 701

${ }^{46} \mathrm{G}$. M. Lilley, "The radiated noise from isotropic turbulence with applica- 702 tions to the theory of jet noise," J. Sound Vib. 190(3), 463-476 (1996). 703

${ }^{47}$ J. T. Stone, R. H. Self, and C. J. Howls, "A complex ray-tracing tool for 704 high-frequency mean field flow-interaction effects in jets," in Proceedings 705 of the 20th AIAA/CEAS Aeroacoustics Conference, AIAA Paper No. 2014- 706 2757 (2014).

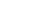

56

67

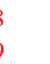

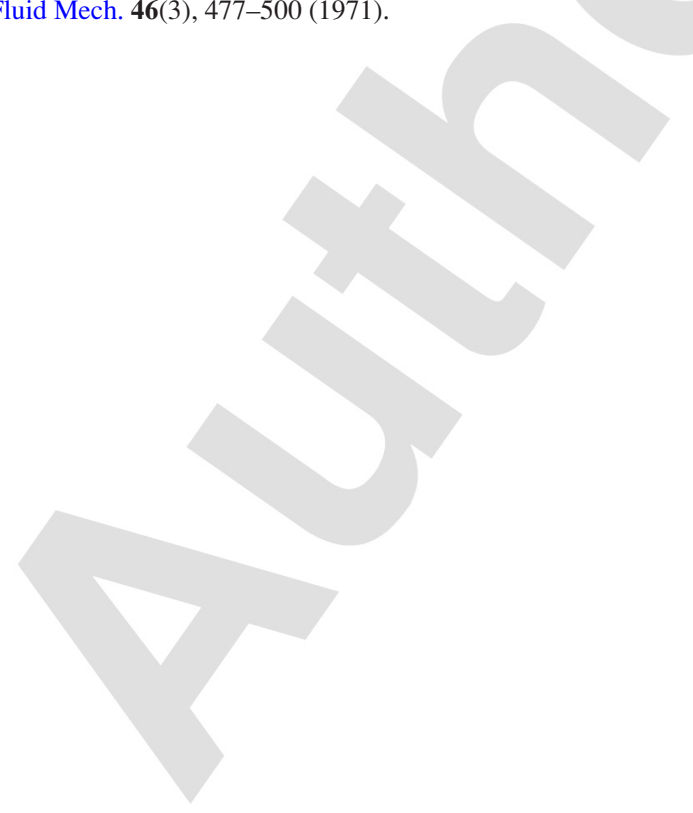

